\title{
Spectral Assessment of Isostatic Gravity Models Against CHAMP, GRACE, GOCE Satellite-Only and Combined Gravity Models
}

\author{
Dimitrios TSOULIS and Konstantinos PATLAKIS \\ Department of Geodesy and Surveying, Aristotle University of Thessaloniki, \\ Thessaloniki, Greece; e-mail: tsoulis@auth.gr
}

\begin{abstract}
The availability of digital elevation databases representing the topographic and bathymetric relief with global homogeneous coverage and increasing resolution permits the computation of crust-related Earth gravity models, the so-called topographic/isostatic Earth gravity models (henceforth T/I models). Although expressing the spherical harmonic content of the topographic masses, the interpretation purpose of T/I models has not been given the attention it deserves, apart from the fact that they express some degree of compensation to the observed spectrum of the topographic heights, depending on the kind of the applied compensation mechanism. The present contribution attempts to improve the interpretation aspects of T/I Earth gravity models. To this end, a rigorous spectral assessment is performed to a standard Airy/Heiskanen T/I model against different CHAllenging Minisatellite Payload (CHAMP), Gravity Recovery and Climate Experiment (GRACE), Gravity field and steadystate Ocean Circulation Explorer (GOCE) satellite-only, and combined gravity models. Different correlation bandwidths emerge for these four groups of satellite-based gravity models. The band-limited forward computation of the models using these bandwidths reproduces nicely the main features of the applied T/I model.
\end{abstract}

Key words: topographic/isostatic gravity models, CHAMP-only models, GRACE-only models, GOCE-only models, combined gravity models. 


\section{INTRODUCTION}

The implementation of the high-low and low-low Satellite-to Satellite Tracking (SST) and Satellite Gravity Gradiometry (SGG) configurations through the corresponding dedicated gravity satellite missions CHAMP (Reigber et al. 2002), GRACE (Tapley et al. 2004), and GOCE (Rummel et al. 2011) has enabled the production of some satellite-only and combined solutions for the Earth's geopotential (Tapley et al. 2004, Flechtner et al. 2010, Pail et al. 2011). Using the perturbed satellite orbit observed by the on-board Global Positioning System (GPS) receiver and quantifying the non-gravitational components through the accelerometer readings, the release of different gravity field models over the last decade or so was made possible, depending on the mathematical model used for the adjustment and the range of the employed satellite data. Furthermore, the combination of these satellite observations with other satellite and terrestrial data led to the computation of socalled combined gravity models. The International Centre for Global Earth Gravity Field Models (ICGEM) Service at the GFZ Helmholtz Centre Potsdam administrates almost all available satellite-only and combined gravity models in terms of their coefficients and their variances, when available. A separate category of gravity models concerns the so-called topographic/ isostatic models. Their special feature lies in the fact that they are linked directly to the geometry of the upper crustal boundary surface, as this is expressed by the topography/bathymetry interface. Their numerical computation can be performed directly from the gridded height values of a global digital elevation model of continents and oceans (terrain and bathymetry), with their maximum degree and order of expansion being limited by the spatial resolution of the elevation database. In terms of their information content, the T/I models express the power spectrum of the topographical masses, thus they include the high and very high frequencies of the observed field. As the satellite-derived models capture mainly the medium-to-long part of the gravity spectrum it is the motivation of the present contribution to investigate the spectral characteristics of a standard T/I model when compared with a selection of the most representative CHAMP-only, GRACE-only, GOCE-only, and combined models. In this way we hope to increase our understanding on the spectral assets of T/I models and quantify their relation with the other available models.

\section{TOPOGRAPHIC/ISOSTATIC GRAVITY MODELS}

The theory of T/I gravity models is based on the definition of the gravitational potential which is generated by the mass balance between crust and mantle. Stimulated by the availability of global digital databases describing the shape and geometry of the Earth's upper crust and the advance of com- 
puter systems permitting the cumbersome task of computing the gravitational signal of these mass anomalies, a number of geodetic papers appeared in the 1980s dealing with both theoretical and numerical aspects of evaluating T/I gravity models. The topic remains open until today with the active interest on T/I models emerging from their unique assets in gravity field modelling and interpretation. One of these assets is the linkage of T/I models with the high-frequency part of the observed field. The maximum degree of expansion of a T/I model is restricted solely by the spatial resolution of the used global elevation model. Since its computation is purely numerical, the denser the input global elevation grid the higher the retrieved frequencies of the gravity field. This characteristic is employed in the evaluation of Earth Gravity Models or the construction of Synthetic Earth Models, both popular applications in current gravity field research.

Two are the most common definitions for the topographic/isostatic potential. Sünkel (1985) defines it as the potential of all mass disturbances, $\delta \rho$, relative to an ideal crustal layer of uniform density, $\rho_{c}$, and thickness, $D$, which are superimposed upon underlying material of uniform density, $\rho_{m}$. Pavlis and Rapp (1990) define the topographic/isostatic potential as the difference between the potential induced by an Earth, which would behave as prescribed by a certain isostatic hypothesis minus a reference potential induced by a simplified Earth model consisting of a crustal layer of uniform density, $\rho_{c}$, and thickness, $D$, superimposed on a denser layer of uniform density, $\rho_{m}$. The two definitions are equivalent, the latter referring to a difference between two potentials and the former to a potential induced by differences in mass, both leading to an identical result. Claessens (2002) proposes a hybrid definition for the topographic/isostatic potential combining the previous two definitions, according to which the T/I potential emerges as the difference between a mass distribution according to a certain isostatic hypothesis and a simplified Earth model consisting of an ideal crustal layer of uniform density, $\rho_{c}$, and thickness, $D$, superimposed on a denser layer of uniform density, $\rho_{m}$.

Crucial to the evaluation of T/I models is the way the lower boundary between crust and mantle is defined, in other words, the way the crustmantle mass equilibrium is approached. The geometry of Moho bounds the integration of the disturbing masses in the radial direction thus defining the numerical values of the obtained T/I spherical harmonic coefficients. Different physical approaches lead to different geometries of the corresponding interface and thus to different mathematical definitions of the T/I coefficients. Applying the Airy/Heiskanen isostatic model, which is the most popular isostatic mechanism used to describe the hydrostatic equilibrium between crust and mantle in the frame of a T/I gravity model, the obtained coefficients are evaluated by the final expressions (Sünkel 1986) 


$$
\left\{\begin{array}{l}
\bar{C}_{l m}^{I} \\
\bar{S}_{l m}^{I}
\end{array}\right\}=\frac{3}{4 \pi \bar{\rho} R} \frac{1}{2 l+1} \iint_{\sigma}\left(A^{T}(Q)-A^{C}(Q)\right) Y_{l m}(Q) d \sigma_{Q},
$$

with $A^{T}$ and $A^{C}$ denoting the surface topography and the compensation part defined respectively as

$$
A^{T}(Q)=\rho_{c r} \int_{r=R}^{R+h}\left(\frac{r_{Q}}{R}\right)^{l+2} d r_{Q},
$$

and

$$
A^{C}(Q)=\Delta \rho \int_{r=R-D-t}^{R-D}\left(\frac{r_{Q}}{R}\right)^{l+2} d r_{Q} .
$$

In the last two expressions, $\rho_{c r}$ and $\Delta \rho$ stand for the constant density of the crust and the constant density contrast between crust and mantle, respectively, and $D$ is the thickness of the crust at zero elevation, also known as compensation depth, i.e., the thickness of the crust beyond which the compensation mechanism for root or anti-root thickness $t$ according to Airy/Heiskanen begins to apply. The rest of the involved parameters include the fully normalized spherical harmonic coefficients of the Airy/Heiskanen topographic/isostatic potential $\bar{C}_{l m}^{I}, \bar{S}_{l m}^{I}$, an approximate value for the Earth's radius $R, \bar{\rho}$ is a mean density value of the Earth's masses, e.g., $5500 \mathrm{~kg} \cdot \mathrm{m}^{-3}, l$ and $m$ denote degree and order, respectively, and finally $Y_{l m}$ stands for the abbreviation

$$
Y_{l m}=\bar{P}_{l m}(\cos \theta)\left\{\begin{array}{l}
\cos m \lambda \\
\sin m \lambda
\end{array}\right.
$$

with $\bar{P}_{l m}$ the fully normalized associated Legendre functions expressed in terms of spherical coordinates co-latitude, $\theta$, and longitude, $\lambda$. Once evaluated, the dimensionless T/I coefficients (1) can be expressed in terms of various gravity field functionals by employing the appropriate eigenvalue in the frame of the so-called Meissl's spectral scheme (Rummel and van Gelderen 1995).

The evaluation of the T/I potential coefficients according to Eq. 1 is performed via a harmonic analysis procedure. Indeed, Eq. 1 expresses the standard scheme of harmonic analysis of a continuous and twice integrable function on the sphere. The evaluation of this equation leads to T/I potential harmonic coefficients, which define up to a certain maximum degree and order the corresponding T/I gravity model. The computation of the emerging 
surface integrals on the sphere in Eq. 1 can be performed either using standard numerical integration techniques, or Fast Fourier algorithms for the sphere. Furthermore, a so-called linear approximation to the surface integration can be applied, which speeds the computations and enables the computation of first, second, and third order terms to the T/I coefficients. Sünkel (1985) provides a thorough review of the theoretical and numerical aspects of computing a T/I model. A number of contributions appeared in the geodetic literature on this topic since, either focusing on the selection of the compensation mechanism and the form of the crust-mantle interface, or in the frame of specific applications, with the construction of an Earth Synthetic Model being the most characteristic one (e.g., Claessens 2002, Bagherbandi and Sjöberg 2012a). The numerical implementation of spherical harmonic analysis is linked with several computational issues that have to be properly addressed, including the way the loss of orthogonality of the Legendre functions due to the latitude sampling is treated and whether the elevation data of a global digital elevation database express point values or mean values valid over the corresponding geographical compartment (Tsoulis 1999). The theory of T/I models permits the investigation and determination of the Moho interface and characteristic structures in the Earth's interior (Bagherbandi and Sjöberg 2011, 2013, Tenzer et al. 2013), or the numerical implementation of different applications in gravity field modelling (Eshagh and Bagherbandi 2011, Novák et al. 2013, Bagherbandi and Sjöberg $2012 \mathrm{~b}$ ). The T/I model that is going to be considered in the present study and will be referred to as simply $\mathrm{T} / \mathrm{I}$ in the sequel, is a T/I gravity model up to degree and order 2160 based on the standard Airy/Heiskanen isostatic hypothesis and the global digital terrain and bathymetry model DTM2002 (Pavlis et al. 2005).

\section{SPECTRAL ASSESSMENT TOOLS OF DIFFERENT GRAVITY MODELS}

Given a certain model there exist different measures for its assessment and evaluation both in the space as well as the coefficients domain. Apart from the definitions arising directly from the availability of the coefficients and variances of one model, e.g., degree variances and error degree variances, other assessment tools exist when a second model is available, acting either as a reference model or as the model against which a spectral comparison is sought. These relative assessment or evaluation tools include Root Mean Square (RMS) anomaly or undulation differences by degree, correlation, smoothing, and percentage difference by degree, gain, or signal-to-noise ratio. These quantities have been used in a routinely fashion in global gravity field modelling and their definition can be found in standard geodetic literature, e.g., Tscherning (1985), Rapp (1986), Lemoine et al. (1998), while they 
are used in simulation or interpretation studies of different available gravity models (Tsoulis and Patlakis 2007), for exploring the relation between topography and Moho in terms of gravity spectra (Martinec 1993), or as statistical tools in the frame of satellite gravity field theory (Sneeuw 2000). We are going to present the definitions of only those quantities that are considered here, the rest of the mathematical definitions can be found in the aforementioned references. It is useful to note, however, that similar definitions that are given with respect to the evolving degree of a model hold for the order as well. Thus, the correlation per degree and order, two quantities which are presented in the following comparisons are defined, respectively, as

$$
\rho_{l}=\frac{\sum_{m=0}^{l}\left(\bar{C}_{l m}^{A} \bar{C}_{l m}^{B}+\bar{S}_{l m}^{A} \bar{S}_{l m}^{B}\right)}{\sqrt{\sigma_{l}^{2}\left(\bar{C}_{l m}^{A}, \bar{S}_{l m}^{A}\right) \sigma_{l}^{2}\left(\bar{C}_{l m}^{B}, \bar{S}_{l m}^{B}\right)}},
$$

and

$$
\rho_{m}=\frac{\sum_{l=m}^{l_{\max }}\left(\bar{C}_{l m}^{A} \bar{C}_{l m}^{B}+\bar{S}_{l m}^{A} \bar{S}_{l m}^{B}\right)}{\sqrt{\sigma_{m}^{2}\left(\bar{C}_{l m}^{A}, \bar{S}_{l m}^{A}\right) \sigma_{m}^{2}\left(\bar{C}_{l m}^{B}, \bar{S}_{l m}^{B}\right)}},
$$

where $\left(\bar{C}_{l m}^{A}, \bar{S}_{l m}^{A}\right)$ and $\left(\bar{C}_{l m}^{B}, \bar{S}_{l m}^{B}\right)$ denote the fully normalized spherical harmonic coefficients of two expansions, symbolically denoted here as models $\mathrm{A}$ and $\mathrm{B}, \sigma_{m}^{2}\left(\bar{C}_{l m}^{A}, \bar{S}_{l m}^{A}\right)$ and $\sigma_{m}^{2}\left(\bar{C}_{l m}^{B}, \bar{S}_{l m}^{B}\right)$ are the order variances corresponding to the two models, expressing cumulative quadratic sums of the respective coefficients for increasing order, $\sigma_{l}^{2}\left(\bar{C}_{l m}^{A}, \bar{S}_{l m}^{A}\right)$ and $\sigma_{l}^{2}\left(\bar{C}_{l m}^{B}, \bar{S}_{l m}^{B}\right)$ are the degree variances. The order-relevant quantities permit the investigation of the zonal characteristics of a given model, e.g., by verifying its alleged asymmetry or non-homogeneity in the longitudinal direction. It is important to note here that the present analysis neglects correlations that are inherent in the different satellite-only gravity solutions and can be quantified through a detailed variance-covariance analysis. As the full variance-covariance information is not always available, we focus only on the information content that can be obtained directly by the coefficients and their variances.

The correlation per degree provides a numerical quantification of a direct comparison between the two models, though it cannot reflect entirely the agreement or disagreement between them. Even if a high correlation by degree exists, the two models may still differ by a dominant scale factor. Therefore, the definition of additional complementary assessment measures is necessary. A better quantification and understanding of the correlations of- 
fered by Eqs. 5 and 6 is obtained from the so-called percentage difference by degree or by order, given, respectively, by the expressions

$$
P_{l}=\left[\frac{\sum_{m=0}^{l}\left(\Delta \bar{C}_{l m}^{2}+\Delta \bar{S}_{l m}^{2}\right)}{\sigma_{l}^{2}\left(\bar{C}_{l m}^{A}, \bar{S}_{l m}^{A}\right)}\right]^{1 / 2} \times 100,
$$

and

$$
P_{m}=\left[\frac{\sum_{l=m}^{l_{\max }}\left(\Delta \bar{C}_{l m}^{2}+\Delta \bar{S}_{l m}^{2}\right)}{\sigma_{m}^{2}\left(\bar{C}_{l m}^{A}, \bar{S}_{l m}^{A}\right)}\right]^{1 / 2} \times 100 .
$$

The percentage difference by degree and order tool defines a quantity expressing the level of smoothing one obtains if model A is subtracted from B (Rapp 1986, Tscherning 1985).

\section{CHAMP, GRACE, GOCE-ONLY AND COMBINED MODELS VERSUS T/I MODELS}

The included figures present some selected quantities validating the aforementioned T/I model with respect to different satellite-only and combined models. The computations are grouped in correspondence to the models against which the comparisons are drawn. Thus, Figs. 1-3 refer to the CHAMP-only models AIUB-CHAMP01S (Prange et al. 2008) and EIGENCHAMP03S (Reigber et al. 2005), Figs. 4-6 present comparisons with the GRACE-only models AIUB-GRACE01S (Jäggi et al. 2010), ITGGRACE03S (Mayer-Gürr 2007), GGM03S (Tapley et al. 2007), and EIGEN5S (Förste et al. 2008), Figs. 7-9 present comparisons with the GOCE-only models SPW-R2 (Migliaccio et al. 2011), DIR-R2 (Bruinsma et al. 2011), and TIM-R3 (Pail et al. 2010), and the final group of figures (Figs. 10-12) demonstrates comparisons with respect to the combined models EIGEN51C (Bruinsma et al. 2010), GGM03C (Tapley et al. 2007), GIF48 (Ries et al. 2011), and EIGEN-6C2 (Förste et al. 2012). For the sake of clarity and in order to facilitate the evaluation of the different kinds of models, the same kind of computations has been repeated for all four groups. First, the relative spectral assessment quantities of correlation per degree and percentage difference by degree have been performed, in order to identify characteristic spectral bandwidths of the evaluated models. Then, according to the rough estimate of bandwidths for which a characteristic correlation could be detected, a band-limited computation of second order radial derivatives at GOCE altitude using this part of the models has been performed. 

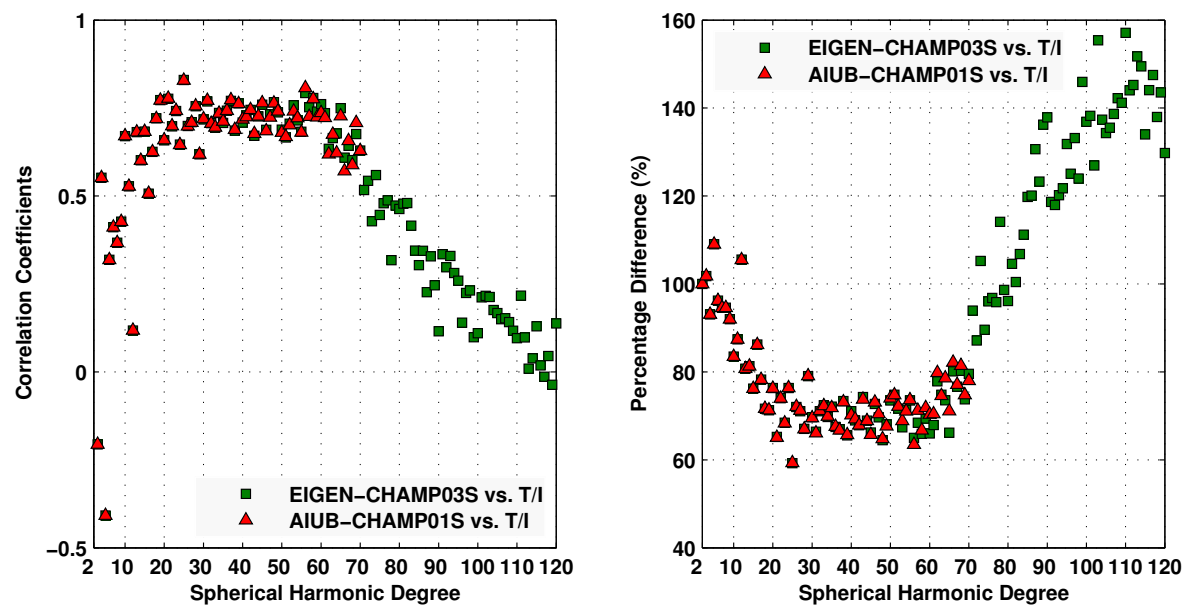

Fig. 1. Correlation per degree (left panel) and percentage difference per degree (right panel) of model T/I with respect to two CHAMP-only models. Colour version of this figure is available in electronic edition only.
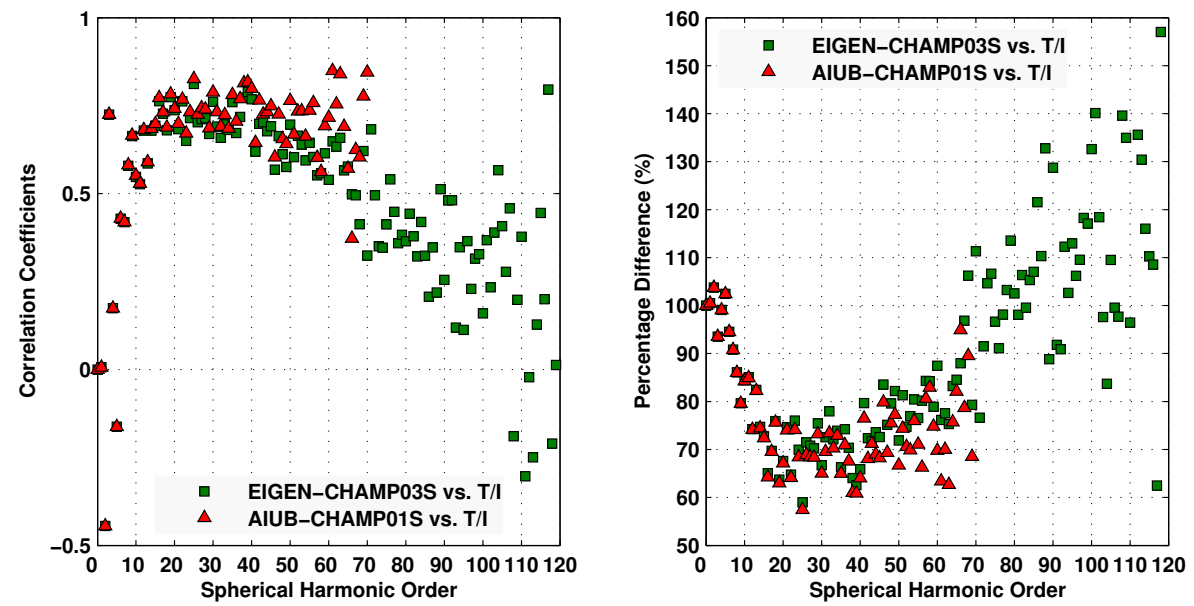

Fig. 2. Correlation per order (left panel) and percentage difference per order (right panel) of model T/I with respect to two CHAMP-only models. Colour version of this figure is available in electronic edition only.

The main outcomes of these comparisons could be briefly summarized as follows:

- The CHAMP-only models reveal the smallest bandwidth of agreement with the T/I model. The agreement bandwidth is located at the degree range $20-60$. 

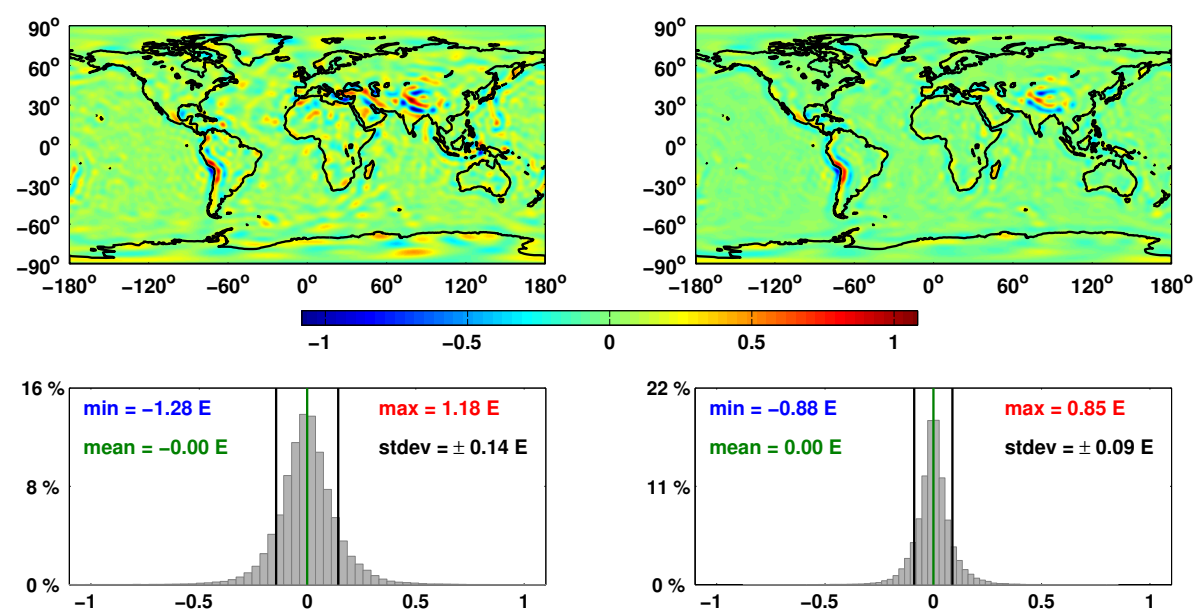

Fig. 3. Second order radial derivative of disturbing potential at $h=250 \mathrm{~km}$ for the spectral bandwidth $20 \leq l \leq 60$, and models AIUB-CHAMP01S (left panel) and T/I (right panel). Unit is Eötvös. Colour version of this figure is available in electronic edition only.
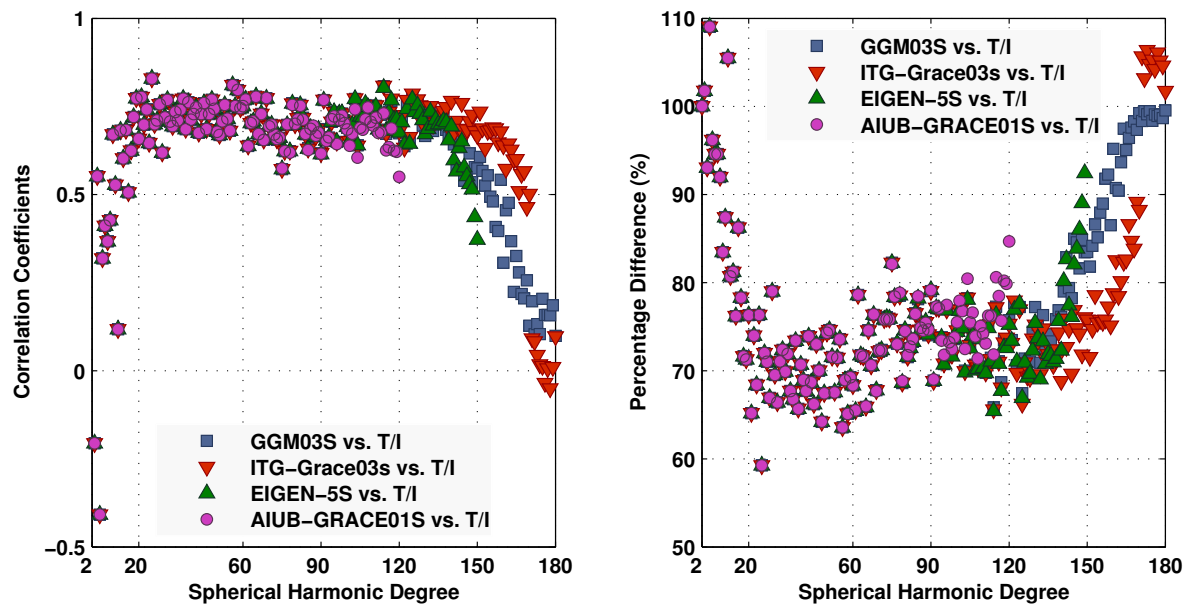

Fig. 4. Correlation per degree (left panel) and percentage difference per degree (right panel) of model T/I with respect to four GRACE-only models. Colour version of this figure is available in electronic edition only.

- GRACE-only models define a wider degree range of agreement with the $\mathrm{T} / \mathrm{I}$ model. The length of the agreement bandwidth varies between 100 and 120 degrees for the different models and is found at the degree range 20-150. However, the agreement is now less constant and it fluctuates between 60 and $80 \%$. 

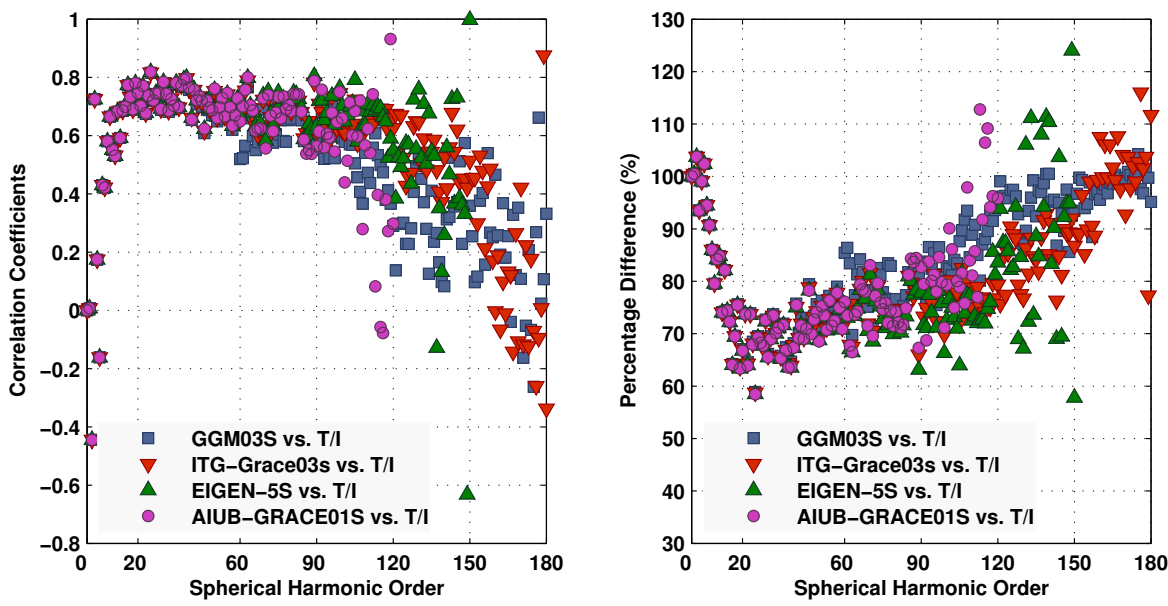

Fig. 5. Correlation per order (left panel) and percentage difference per order (right panel) of model T/I with respect to four GRACE-only models. Colour version of this figure is available in electronic edition only.
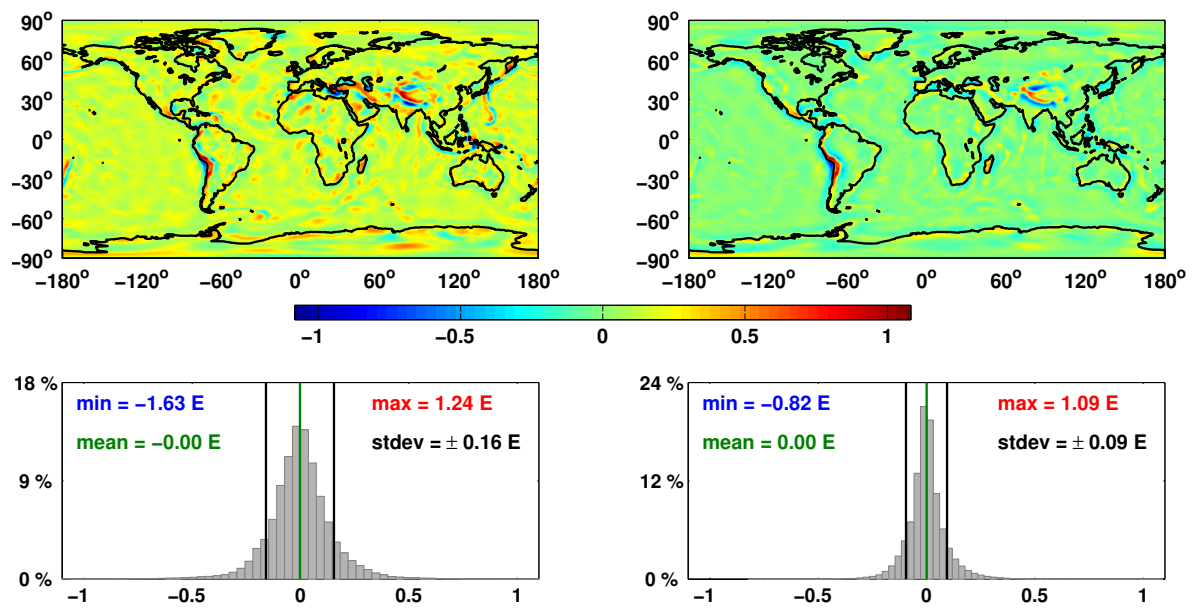

Fig. 6. Second order radial derivative of disturbing potential at $h=250 \mathrm{~km}$ for the spectral bandwidth $20 \leq l \leq 150$, and models ITG-GRACE03 (left panel) and T/I (right panel). Unit is Eötvös. Colour version of this figure is available in electronic edition only.

- The GOCE-only models display by far the highest degree of correlation with T/I for a bandwidth ranging from 20 to 200. The upper bound of this bandwidth varies between the different GOCE models, reaching up to degree 220. Also the correlation patterns of the different GOCE models 

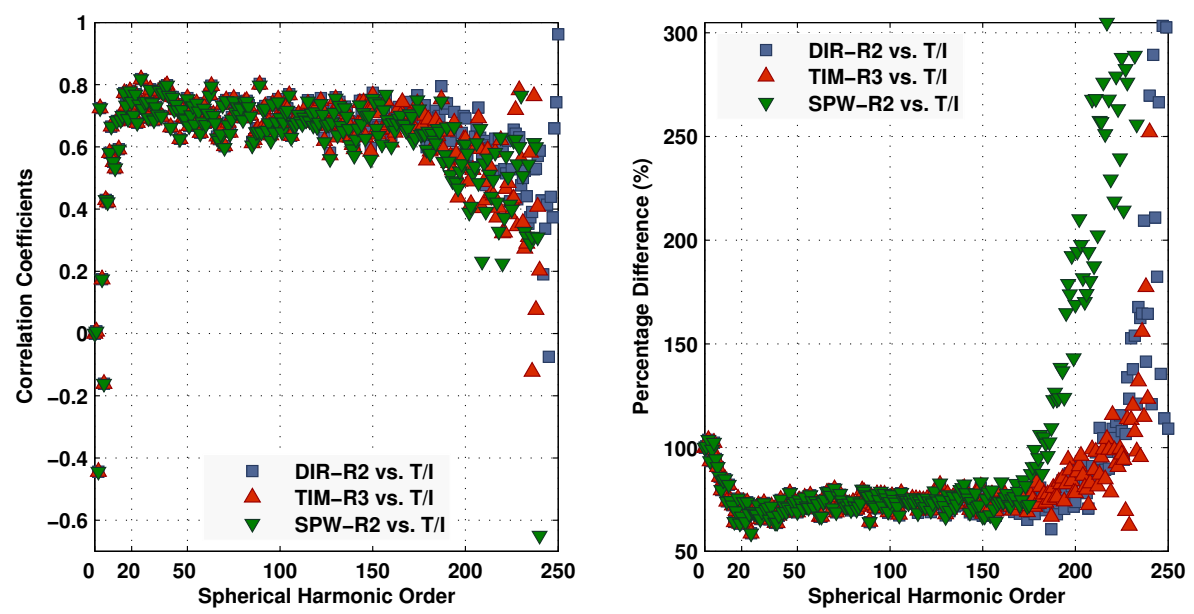

Fig. 7. Correlation per degree (left panel) and percentage difference per degree (right panel) of model T/I with respect to three GOCE-only models. Colour version of this figure is available in electronic edition only.
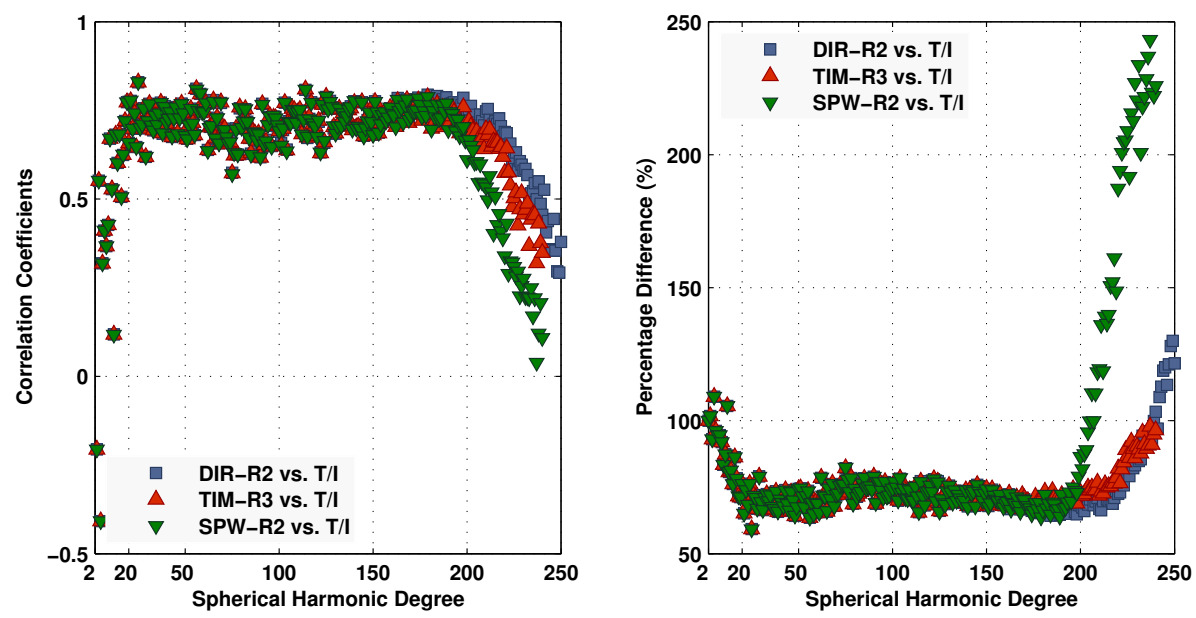

Fig. 8. Correlation per order (left panel) and percentage difference per order (right panel) of model T/I with respect to three GOCE-only models. Colour version of this figure is available in electronic edition only.

with respect to T/I are very similar. The short-wavelength sensitivity of the GOCE models seems to be apparent in these comparisons. The link of the gradiometry observations to upper crust anomaly structures is nicely demonstrated in the correlations of the GOCE only models with the corresponding topography related models. 

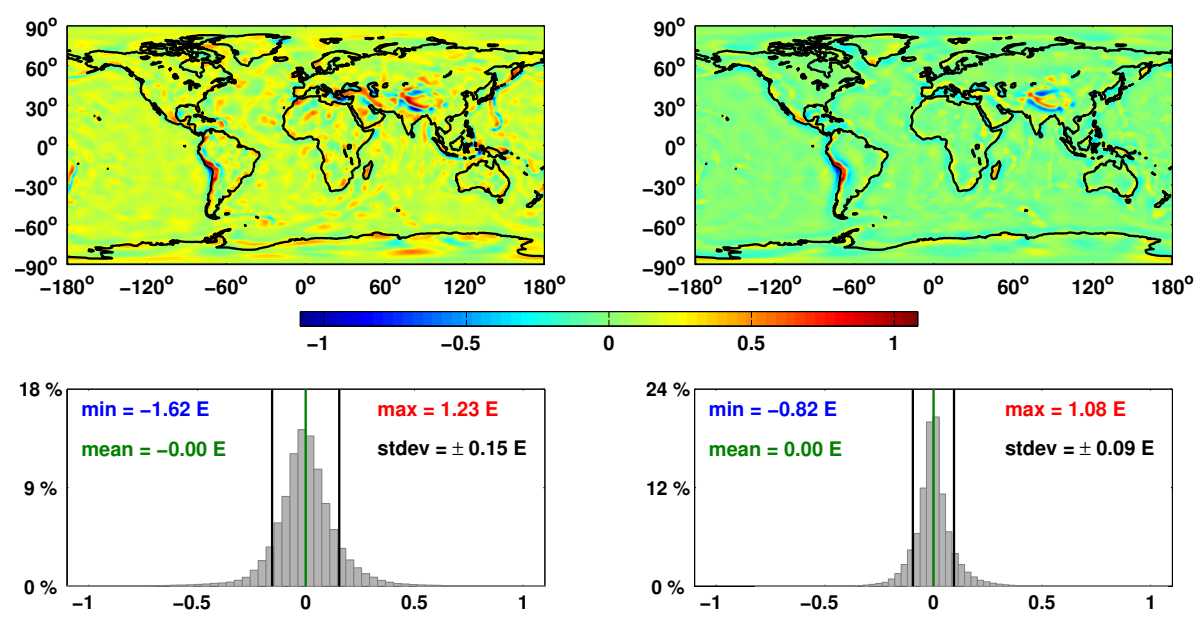

Fig. 9. Second order radial derivative of disturbing potential at $h=250 \mathrm{~km}$ for the spectral bandwidth $20 \leq l \leq 200$, and models SPW-R2 (left panel) and T/I (right panel). Unit is Eötvös. Colour version of this figure is available in electronic edition only.
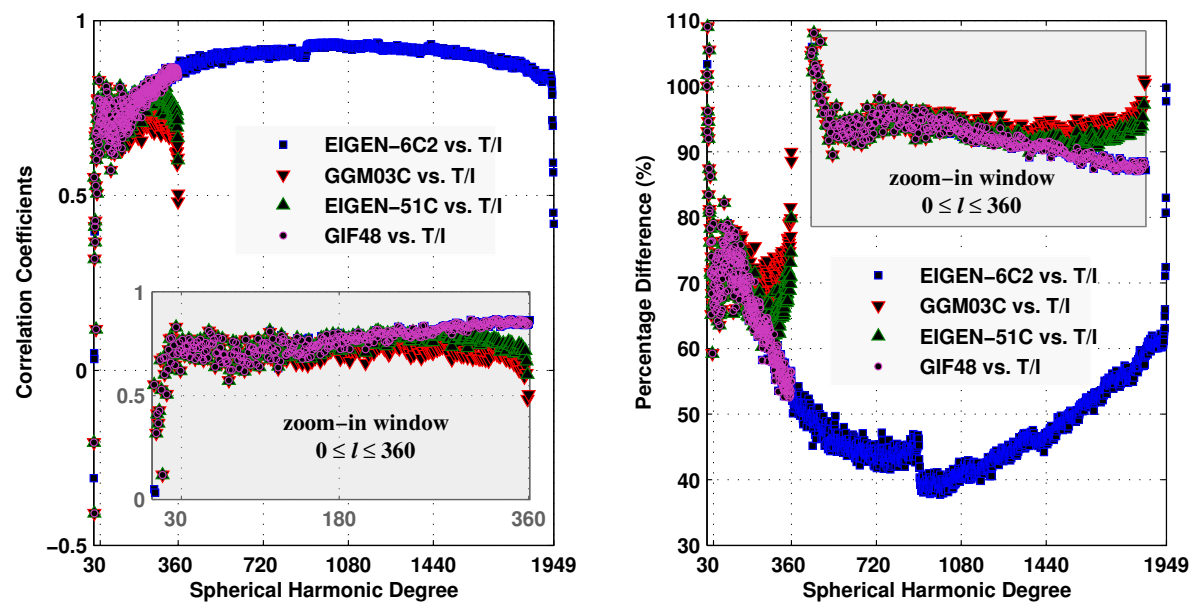

Fig. 10. Correlation per degree (left panel) and percentage difference per degree (right panel) of model T/I with respect to four combined models. Colour version of this figure is available in electronic edition only.

- The combined models appear to be the most correlated with the T/I model from all four groups. The correlation bandwidth spans over almost the whole spectrum, representing a very extended window of the medium to high frequencies of the observed spectrum. These spectral patterns are 

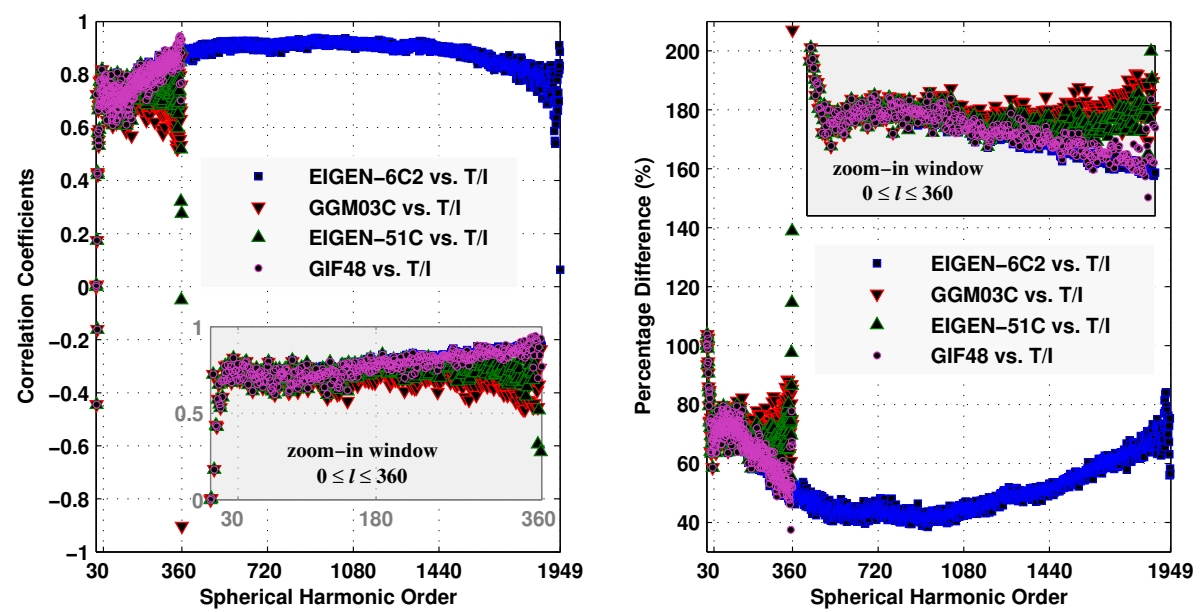

Fig. 11. Correlation per order (left panel) and percentage difference per order (right panel) of model T/I with respect to four combined models. Colour version of this figure is available in electronic edition only.
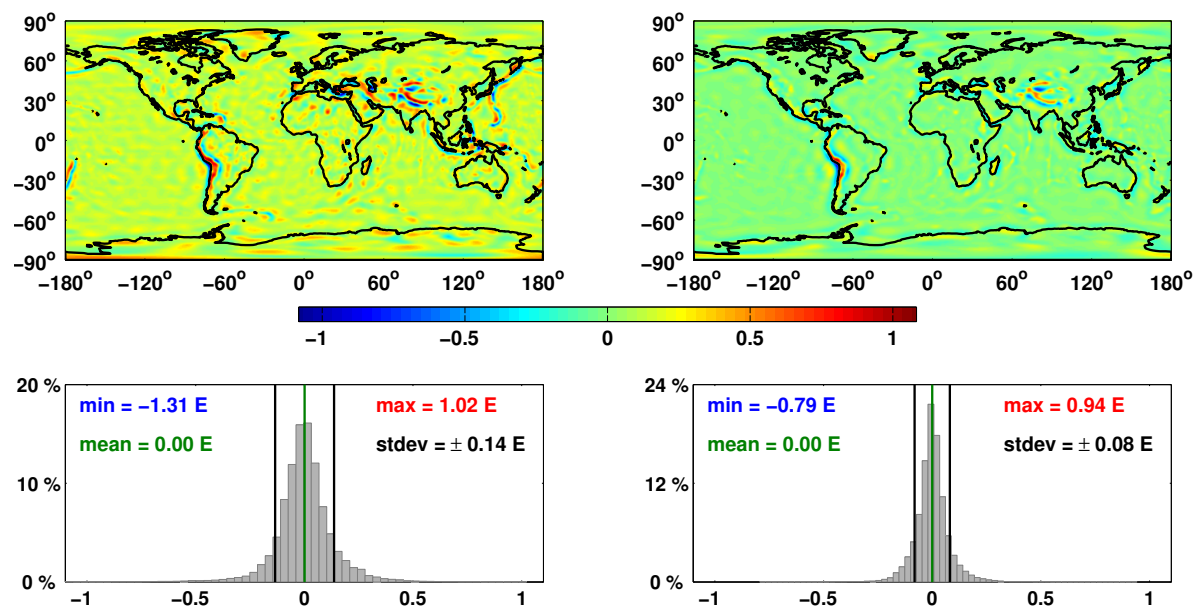

Fig. 12. Second order radial derivative of disturbing potential at $h=250 \mathrm{~km}$ for the spectral bandwidth $30 \leq l \leq 1940$, and models EIGEN-6C2 (left panel) and T/I (right panel). Unit is Eötvös. Colour version of this figure is available in electronic edition only.

expected, since combined models include also terrestrial data, which are highly correlated with the topography-related gravity signal.

- The correlation per order patterns in all four groups appear to be similar to those obtained for correlation per degree, despite the limited number of 
involved coefficients corresponding to a specific order due to the applied truncation.

- In all cases the forward computation of the band-limited T/I model reproduces very accurately both in terms of size as well as lateral variations the features obtained by the satellite-only or combined gravity models.

The above spectral observations become more obvious when transformed in the spatial domain as gravity field functionals, after the appropriate eigenvalue has been taken into account. Since direct gravity gradient observations are now available as GOCE gradiometry data, the computation of second order radial derivatives at a $h=250 \mathrm{~km}$ altitude has been selected for the performed synthesis. Figures 3, 6, 9, and 12 present the forward computation of global grids of second order radial derivatives of the disturbing potential at GOCE altitude using the correlation bandwidth for models AIUB-CHAMP01S, ITG-GRACE03S, GOCE-SPW-R2, and EIGEN-6C2 respectively that emerged from our previous analysis. These band-limited computations are compared directly with the corresponding bandwidth of the T/I model. All four truncated versions of T/I manage to capture the main features of $T_{r r}$ at GOCE altitude. AIUB-CHAMP01S offers the most narrow range of values compared with the other satellite-only and combined models. This is expected, as CHAMP models are the least sensitive gravity models to high-frequency characteristics of the observed field. The T/I cannot resolve the whole range of the obtained gravity gradient values for any of the considered models, since all used correlation bandwidths are close to the actual resolution of the models. Thus, a small amount of coefficients is truncated from these complete solutions in comparison to the large amount of missing information expressed by the T/I truncated versions. Combination model EIGEN-6C2 produces the most detailed lateral information pattern for $T_{r r}$ at GOCE altitude. The high solvable degree of 1949 combined with the employment of EGM2008, especially for degree 370 and onwards, and the inclusion of GOCE data in its compilation lead to the most detailed synthetic field of gravity gradients from all considered models.

The performed analysis carries out a quantification of the general assets of CHAMP, GRACE, GOCE, and combined related models in terms of their accuracy, wavelength characteristics, and overall performance. The bounded degree and order ranges that are obtained from the present comparisons define in detail those bandwidths of these models where a documented correlation with a T/I model is observed. Since the T/I model represents a unique case of gravity field model which contains due to its origin and algorithmic procedure based on global elevation data the high and very high frequencies of the observed gravity field, it offers an ideal independent assessment tool for the different available gravity models. 
The increased bandwidth of agreement with the T/I model in the case of GRACE, GOCE, and combined models is a nice demonstration of how the increased accuracy of primary low-low SST and SGG observations leads to a more accurate gravity field that assimilates better the high-frequency constituents of the gravity field that its predecessor CHAMP. This becomes even more apparent in the case of the combined models, where GRACE data have been used to a great extent in the compilation process of these models.

\section{CONCLUDING REMARKS}

The computation of T/I gravity models, a geodetic procedure which utilizes the geometry of the crustal masses as this, is obtained from a global elevation model, gains currently again in significance as an abundance of new satellite-only and combined Earth gravity models becomes available from the analysis of precise tracking, accelerometry and gradiometry data of different satellites or satellite constellations. The T/I models offer an independent reference model, which can be used in order to assess uniformly the different available models and identify and quantify their characteristic spectral bandwidths. The application of relative spectral tools used in the present study revealed different correlation patterns between an Airy/Heiskanen type T/I model full to degree and order 2160 and different CHAMP-only, GRACE-only, GOCE-only, and combined gravity models. The T/I model shows the most profound correlation pattern with respect to the combined models, manifesting the dependence of the latter on ground data including global terrain and bathymetry data. In all performed calculations the correlation patterns concern both the degrees and orders, which permits the quantification of the level of homogeneity of a single model. The obtained correlation bandwidths of the T/I model perform extremely well in reproducing the main features of the satellite-only and combined models, when transformed in gravity field contributions in the space domain.

Using the T/I model as the reference model for our comparative analyses we decided to focus on the correlation bandwidths that emerge from the spectral assessment procedure we applied to the different satellite-only and combined models. The band-limited spatial computations of second order radial derivatives using models AIUB-CHAMP01S, ITG-GRACE03S, GOCE-SPW-R2, and EIGEN-6C2 in direct comparison with model T/I, using those spectral bandwidths that emerged from the performed spectral comparisons verify nicely how the specific bandwidths of the satellite-only or combined models capture in an overall sense the main features of a T/I model, which include, e.g., the effect of smoothing the observed field without any loss of physical information that any low-pass filtering would cause (Göttl and Rummel 2009). Furthermore, using these specific truncated versions of these models we managed to draw a more detailed comparative as- 
sessment of the different models against T/I. Thus, the combined model EIGEN-6C2 presents the highest level of accordance in terms of both degree and order-wise spectral correlation patterns as well as the band-limited spatial computations of second order radial derivatives at GOCE altitude. GOCE model SPW-R2 presents the second best performance in terms of bandwidth range of the obtained correlations and range of values and lateral variations in the second order derivatives computed fields. The GRACE model ITG-GRACE03S is slightly worse than SPW-R2, although performing in the same order of magnitude of all involved spectral and spatial comparisons. CHAMP-only model AIUB-CHAMP01S proves to be the worst candidate when it comes to seek the terrain-related gravity field information in a complete gravity field solution. It presents the least spectral level of agreement with T/I, while it fails to reconstruct the spatial field of gravity gradients with the detail of T/I. Both the different absolute values of solvable degrees as well as the different accuracy and resolution levels inherited in the different satellite-only methodologies are revealed in these comparisons.

Acknowledgments. The International Centre for Global Earth Models (ICGEM) at the Geoforschungszentrum Potsdam (GFZ-Potsdam) maintains a freely accessible database of almost all models used in the present survey at http://icgem.gfz-potsdam.de/ICGEM. Financial support through European Space Agency (ESA) contract 22316/09/NL/CBI is highly appreciated. Finally, the constructive comments by Dr. Eshagh and an anonymous reviewer are gratefully acknowledged.

\section{References}

Bagherbandi, M., and L.E. Sjöberg (2011), Comparison of crustal thickness from two gravimetric-isostatic models and CRUST2.0, Stud. Geophys. Geod. 55, 4, 641-666, DOI: 10.1007/s11200-010-9030-0.

Bagherbandi, M., and L.E. Sjöberg (2012a), A synthetic Earth gravity model based on a topographic-isostatic model, Stud. Geophys. Geod. 56, 4, 935-955, DOI: $10.1007 / \mathrm{s} 11200-011-9045-1$.

Bagherbandi, M., and L.E. Sjöberg (2012b), Modelling the density contrast and depth of the Moho discontinuity by seismic and gravimetric-isostatic methods with an application to Africa, J. Afr. Earth Sci. 68, 111-120, DOI: 10.1016/j.jafrearsci.2012.04.003.

Bagherbandi, M., and L.E. Sjöberg (2013), Improving gravimetric-isostatic models of crustal depth by correcting for non-isostatic effects and using 
CRUST2.0, Earth-Sci. Rev. 117, 29-39, DOI: 10.1016/j.earscirev.2012. 12.002 .

Bruinsma, S.L., J.-C. Marty, G. Balmino, R. Biancale, Ch. Förste, O. Abrikosov, and H. Neumayer (2010), GOCE gravity field recovery by means of the direct numerical method. In: H. Lacoste-Francis (ed.), Proc. ESA Living Planet Symposium, 27 June - 2 July 2010, Bergen, Norway, Vol. 27, European Space Agency, Publication SP-686.

Bruinsma, S., J.-C. Marty, G. Balmino, Ch. Förste, O. Abrikosov, and H. Neumayer (2011), A GOCE-only gravity field model inferred from 6.7 months of data using the direct numerical method, Geophys. Res. Abstr. 13, EGU20115850 .

Claessens, S.J. (2002), A synthetic Earth model analysis, implementation, validation and application, M.Sc. Thesis, Delft University of Technology, Faculty of Civil Engineering and Geosciences, Department of Geodesy, Delft, The Netherlands, 75 pp.

Eshagh, M., and M. Bagherbandi (2011), Smoothing impact of isostatic crustal thickness models on local integral inversion of satellite gravity gradiometry data, Acta Geophys. 59, 5, 891-906, DOI: 10.2478/s11600-011-0017-1.

Flechtner, F., Ch. Dahle, K.H. Neumayer, R. König, and Ch. Förste (2010), The Release 04 CHAMP and GRACE EIGEN gravity field models. In: F.M. Flechtner, Th. Gruber, A. Güntner, M. Mandea, M. Rothacher, T. Schöne, and J. Wickert (eds.), System Earth via Geodetic-Geophysical Space Techniques, Advanced Technologies in Earth Sciences, Springer, Berlin Heidelberg, 41-58, DOI: 10.1007/978-3-642-10228-8_4.

Förste, Ch., F. Flechtner, R. Schmidt, R. Stubenvoll, M. Rothacher, J. Kusche, H. Neumayer, R. Biancale, J.-M. Lemoine, F. Barthelmes, S. Bruinsma, R. König, and Ul. Meyer (2008), EIGEN-GL05C - A new global combined high-resolution GRACE-based gravity field model of the GFZ-GRGS cooperation, Geophys. Res. Abstr. 10, EGU2008-A-03426.

Förste, Ch., R. Shako, F. Flechtner, C. Dahle, O. Abrikosov, K.-H. Neumayer, F. Barthelmes, R. König, S.-L. Bruinsma, J.-C. Marty, J.-M. Lemoine, G. Balmino, and R. Biancale (2012), A new release for EIGEN-6 - The latest combined global gravity field model including LAGEOS, GRACE and GOCE data from the collaboration of GFZ Potsdam and GRGS Toulouse, Geophys. Res. Abstr. 14, EGU2012-2821.

Göttl, F., and R. Rummel (2009), A geodetic view on isostatic models, Pure Appl. geophys. 166, 8-9, 1247-1260, DOI: 10.1007/s00024-004-0489-x.

Jäggi, A., G. Beutler, and L. Mervart (2010), GRACE gravity field determination using the Celestial Mechanics Approach - first results. In: S.P. Mertikas (ed.), Gravity, Geoid and Earth Observation, International Association of Geodesy Symposia, Vol. 135, Springer, Berlin Heidelberg, 177-184, DOI: 10.1007/978-3-642-10634-7_24. 
Lemoine, F.G., S.C. Kenyon, J.K. Factor, R.G. Trimmer, N.K. Pavlis, D.S. Chinn, C.M. Cox, S.M. Klosko, S.B. Luthcke, M.H. Torrence, Y.M. Wang, R.G. Williamson, E.C. Pavlis, T.R. Olson, and R.H. Rapp (1998), The development of the joint NASA GSFC and the National Imagery and Mapping Agency (NIMA) geopotential model EGM96, NASA/TP-1998206861, National Aeronautics and Space Administration, Goddard Space Flight Center, Greenbelt, USA.

Martinec, Z. (1993), A model of compensation of topographic masses, Surv. Geophys. 14, 4-5, 525-535, DOI: 10.1007/BF00690575.

Mayer-Gürr, T. (2007), ITG-Grace03s: The latest GRACE gravity field solution computed in Bonn. In: Joint International GSTM and DFG SPP Symposium, 15-17 October 2007, Potsdam.

Migliaccio, F., M. Reguzzoni, A. Gatti, F. Sansò, and M. Herceg (2011), A GOCEonly global gravity field model by the space-wise approach, Geophys. Res. Abstr. 13, EGU2011-10063-3.

Novák, P., R. Tenzer, M. Eshagh, and M. Bagherbandi (2013), Evaluation of gravitational gradients generated by Earth's crustal structures, Comput. Geosci. 51, 22-33, DOI: 10.1016/j.cageo.2012.08.006.

Pail, R., H. Goiginger, R. Mayrhofer, W.-D. Schuh, J.M. Brockmann, I. Krasbutter, E. Höck, and T. Fecher (2010), GOCE gravity field model derived from orbit and gradiometry data applying the time-wise method. In: H. LacosteFrancis (ed.), Proc. ESA Living Planet Symposium, 27 June - 2 July 2010, Bergen, Norway, Vol. 27, European Space Agency, Publication SP-686.

Pail, R., S. Bruinsma, F. Migliaccio, Ch. Förste, H. Goiginger, W.-D. Schuh, E. Höck, M. Reguzzoni, J.M. Brockmann, O. Abrikosov, M. Veicherts, T. Fecher, R. Mayrhofer, I. Krasbutter, F. Sansò, and C.C. Tscherning (2011), First GOCE gravity field models derived by three different approaches, J. Geod. 85, 11, 819-843, DOI: 10.1007/s00190-011-0467-x.

Pavlis, N.K., and R.H. Rapp (1990), The development of an isostatic gravitational model to degree 360 and its use in global gravity modelling, Geophys. $J$. Int. 100, 3, 369-378, DOI: 10.1111/j.1365-246X.1990.tb00691.x.

Pavlis, N.K., S.A. Holmes, S.C. Kenyon, D. Schmidt, and R. Trimmer (2005), A preliminary gravitational model to degree 2160. In: C. Jekeli, L. Bastos, and J. Fernandes (eds.), Gravity, Geoid and Space Missions, International Association of Geodesy Symposia, Vol. 129, Springer, Berlin Heidelberg, 18-23, DOI: 10.1007/3-540-26932-0_4.

Prange, L., A. Jäggi, G. Beutler, R. Dach, and L. Mervart (2008), Gravity field determination at the AIUB - the Celectial Mechanics Approach. In: M.G. Sideris (ed.), Observing our Changing Earth, International Association of Geodesy Symposia, Vol. 133, Springer, Berlin Heidelberg, 353-362, DOI: 10.1007/978-3-540-85426-5_42.

Rapp, R.H. (1986), Global geopotential solutions. In: H. Sünkel (ed.), Mathematical and Numerical Techniques in Physical Geodesy, Lecture Notes in Earth 
Sciences, Vol. 7, Springer, Berlin Heidelberg, 365-415, DOI: 10.1007/ BFb0010136.

Reigber Ch., H. Lühr, and P. Schwintzer (2002), CHAMP mission status, $A d v$. Space Res. 30, 2, 129-134, DOI: 10.1016/S0273-1177(02)00276-4.

Reigber, Ch., H. Jochmann, J. Wünsch, S. Petrovic, P. Schwintzer, F. Barthelmes, K.-H. Neumayer, R. König, Ch. Förste, G. Balmino, R. Biancale, J.-M. Lemoine, S. Loyer, and F. Perosanz (2005), Earth gravity field and seasonal variability from CHAMP. In: Ch. Reigber, H. Lühr, P. Schwintzer, and J. Wickert (eds.), Earth Observation with CHAMP - Results from Three Years in Orbit, Springer, Berlin Heidelberg, 25-30, DOI: 10.1007/3540-26800-6_4.

Ries, J.C., S. Bettadpur, S. Poole, and T. Richter (2011), Mean background gravity fields for GRACE processing, presented at the GRACE Science Team Meeting, Austin, TX, 8-10 August 2011, ftp://ftp.csr.utexas.edu/pub/grace/ GIF48/GSTM2011_Ries_etal.pdf.

Rummel, R., and M. Vangelderen (1995), Meissl scheme - spectral characteristics of physical geodesy, Manuscr. Geod. 20, 5, 379-385.

Rummel, R., W. Yi, and C. Stummer (2011), GOCE gravitational gradiometry, J. Geod. 85, 11, 777-790, DOI: 10.1007/s00190-011-0500-0.

Sneeuw, N.J. (2000), A semi-analytical approach to gravity field analysis from satellite observations, Ph.D. Thesis, Deutsche Geodätische Kommission, Reihe C, Heft 527, Verlag der Bayerischen Akademie der Wissenschaften, München, $117 \mathrm{pp}$.

Sünkel, H. (1985), An isostatic Earth model, Tech. Rep. No. 367, Department of Geodetic Science and Surveying, The Ohio State University, Columbus, USA, 53 pp.

Sünkel, H. (1986), Global topographic-isostatic models. In: H. Sünkel (ed.), Mathematical and Numerical Techniques in Physical Geodesy, Lecture Notes in Earth Sciences, Vol. 7, Springer, Berlin Heidelberg, 417-462, DOI: $10.1007 / \mathrm{BFb} 0010137$.

Tapley, B.D., S. Bettadpur, M. Watkins, and Ch. Reigber (2004), The gravity recovery and climate experiment: Mission overview and early results, Geophys. Res. Lett. 31, 9, L09607, DOI: 10.1029/2004GL019920.

Tapley, B.D., J. Ries, S. Bettadpur, D. Chambers, M. Cheng, F. Condi, and S. Poole (2007), The GGM03 mean earth gravity model from GRACE, Eos Trans. $A G U$ 88, 52, Fall Meeting Suppl., Abstr. G42A-03.

Tenzer, R., M. Bagherbandi, and P. Vajda (2013), Global model of the upper mantle lateral density structure based on combining seismic and isostatic models, Geosci. J. 17, 1, 65-73, DOI: 10.1007/s12303-013-0009-z.

Tscherning, C.C. (1985), On the long-wavelength correlation between gravity and topography. In: H. Kautzleben (ed.), Proc. 5th Inter. Symposium "Geodesy and Physics of the Earth”, 23-29 September 1984, Magdeburg, G.D.R., 
Veröffentlichungen des Zentralinstituts für Physik der Erde, Vol. 81, 2, Akademie der Wissenschaften der DDR, Potsdam, 134-142.

Tsoulis, D. (1999), Spherical harmonic computations with topographic/isostatic coefficients, Reports in the series IAPG/FESG, Rep. No. 3, Institute of Astronomical and Physical Geodesy, Technical University of Munich, $33 \mathrm{pp}$.

Tsoulis, D., and K. Patlakis (2007), Spectral assessment of recently released CHAMP and GRACE satellite-only Earth gravity models. In: A. Kılıçoğlu, and R. Forsberg (eds.), Proc. 1st Inter. Symp. of the Int. Gravity Field Service "Gravity Field of the Earth", Harita Dergisi, Sp. Issue 18, General Command of Mapping, Ankara, Turkey, 175-180.

Received 21 December 2012

Received in revised form 23 July 2013

Accepted 1 August 2013 